\title{
A162 ENHANCED EXPRESSION OF EPHRINS AND THROMBOSPONDINS IN THE DERMIS OF PATIENTS WITH EARLY DIFFUSE SYSTEMIC SCLEROSIS
}

J Avouac, ${ }^{1,2}$ M Clemessy, ${ }^{3}$ J M Gasc, ${ }^{3}$ B Ruiz, ${ }^{2}$ M C Vacher-Lavenu, ${ }^{4}$ J Wipff, ${ }^{1,2}$ A Kahan, ${ }^{1}$ C Boileau, 2,5 P Corvol, ${ }^{3}$ Y Allanore ${ }^{1,2}{ }^{1}$ Paris Descartes University, Rheumatology A Department, Cochin Hospital, APHP, Paris; ${ }^{2}$ INSERM U781, Necker Hospital, Paris; 3INSERM U833 and Collège de France, Paris, France; ${ }^{4}$ Paris Descartes University, Pathophysiology Department, Cochin Hospital, Paris, ${ }^{5}$ U.V.S. 0 University, Biochemistry, Hormonology and Molecular Genetics Department, Ambroise Paré Hospital, AP-HP, Boulogne, France

10.1136/ard.2010.129650m

Objective Ephrins have emerged as essential regulators of angiogenesis and thrombospondins are inhibitors of angiogenesis and strong promoters of fibrosis that are both involved in systemic sclerosis (SSc). Our aim was to analyse the expression of ephrins and thrombospondins concomitant to VEGF pathway assessment in skin specimens and dermal cultured fibroblasts from patients with SSc.

Methods Skin biopsies were obtained from 8 patients with early diffuse cutaneous subset. In all patients, biopsies were taken from both clinically involved and non-involved skin. Controls consisted of biopsies taken from 4 healthy volunteers matched for age and sex with SSc patients. We analysed the expression of VEGF and its receptors, ephrins (EphB4 and EphrinB2) and thrombospondins (TSP-1 and 2) mRNA by in situ hybridisation in skin specimens from SSc patients compared with controls. We also assessed EphB4/EphrinB2 and TSP-1/2 mRNA expression in cultured dermal fibroblasts from clinically involved and non-involved skin biopsies taken from patients and controls by RT-PCR in basal conditions and after $8 \mathrm{~h}$ of hypoxia.

Results VEGF, VEGFR-1 and EphrinB2 mRNA were upregulated in SSc skin biopsies taken from clinically affected skin (2-, 7- and 3-fold increase, respectively) and non-affected skin (2-, 7- and 3-fold increase, respectively) compared with controls, contrasting with VEGFR-2 and EphB4 which was upregulated only in affected skin (3- and 5-fold increase, respectively). EphrinB2 and EPhB4 mRNA levels were low in control and SSc cultured dermal fibroblasts, suggesting that they do not produce ephrins. TSP- 1 and 2 were upregulated in SSc skin biopsies taken from both involved skin (2- and 3-fold increase, respectively) and non-involved skin (2- and 3-fold increase, respectively). TSP-1 and 2 mRNA levels were also overexpressed in SSc cultured dermal fibroblasts from clinically involved and non-involved skin in a hypoxia independent manner.

Conclusion We have investigated for the first time the expression of EphB4/EphrinB2 and TSP-1/TSP-2 expression in the skin of patients with SSc. EphB4 and EphrinB2 are upregulated in clinically involved skin of SSc patients, suggesting their participation in SSc perturbed angiogenesis. TSP-1/2 are upregulated in both clinically involved and non-involved SSc skin through an overproduction by dermal fibroblasts. These results support the possible intervention of TSPs in the vasculopathy and fibrotic process of SSc. Further studies are now warranted to assess the role of TSP-1 and TSP-2 in SSc in the early activation of transforming growth factor $\beta$ (TGF $\beta$ ) and in the assembly of extracellular matrix proteins accumulated by the overproduction of TGF $\beta$. 